\title{
Hemangioendotelioma kaposiforme torácico con respuesta favorable a sirolimus. Reporte de caso y revisión de la literatura
}

\section{Thoracic kaposiform hemangioendothelioma with a good response to syrolimus. Case report and literature review}

Correspondencia José Tantaleán D.

tantaleanjose@hotmail.com jtantalean@insn.gob.pe

Recibido: 03/10/2018

Arbitrado por pares

Aprobado: 27/03/2019

Citar como: Guerrero-Padilla D, Tantaleán-Da Fieno J, VelásquezValderrama F, León-Paredes $R$. Hemangioendotelioma kaposiforme torácico con respuesta favorable a sirolimus. Reporte de caso y revisión de la literatura. Acta Med Peru. 2019;36(2):124-8

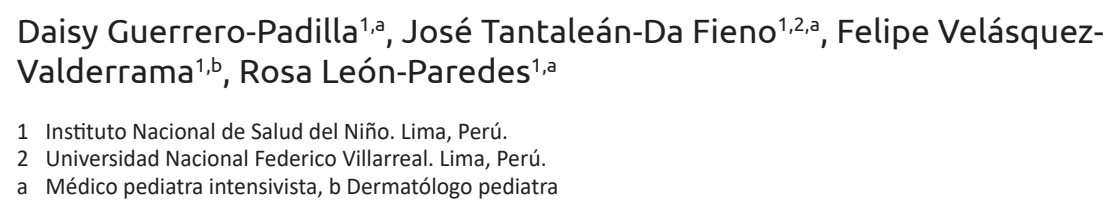

\section{RESUMEN}

Se presenta el caso de una niña de 4 meses de edad que ingresa a la Unidad de Cuidados Intensivos (UCI) por presentar el Síndrome de Kasabach-Merritt asociado a un hemangioendotelioma kaposiforme. Luego de un mes de recibir tratamiento con vincristina, corticoides, propanolol y ácido acetilsalicílico, continúa con evolución desfavorable, incrementándose el tamaño de la lesión. Los exámenes de imágenes (tomografia axial computarizada y resonancia magnética nuclear) muestran compromiso de músculos intercostales, pleura, parénquima pulmonar y trombosis de venas cervicales. Posteriormente, llega a presentar cianosis y dificultad respiratoria por obstrucción de vías aéreas, requiriendo intubación y ventilación mecánica. Se inicia sirolimus, y en la segunda semana de tratamiento se evidencia mejoría de la lesión y de la analítica. Se brinda el alta hospitalaria a los 42 días de terapia con sirolimus, tratamiento que continúa recibiendo hasta un año después.

Palabras clave: Hemangioendotelioma; Invasividad neoplásica; Sirolimus (fuente: DeCS BIREME)

\section{ABSTRACT}

A 4-month girl was admitted to Pediatric Intensive Care Unit (PICU) due to a Kasabach-Merritt Syndrome associated to Kaposiform hemangioendothelioma. After 1-month of treatment with vincristine, steroids, propranolol, and acetylsalicylic acid; her progress was not good, and lesions became larger. Imaging studies (computed tomography and nuclear magnetic resonance) showed involvement of intercostal muscles, pleura, lung parenchyma, and cervical venous thrombosis. Afterwards, the patient developed cyanosis and dyspnea due to an upper airway obstruction, requiring intubation and ventilation support. Sirolimus was started and, on the second week of treatment, both lesion and laboratory parameters improved. The patient was discharged after 42 days of sirolimus therapy. One year later, she is still receiving this drug.

Keywords: Hemangioendothelioma; Neoplasm invasiveness; Sirolimus (source: NLM MeSH). 


\section{INTRODUCCIÓN}

El hemangioendotelioma kaposiforme (HEK) es un tumor vascular potencialmente letal, cuya incidencia es de aproximadamente 0,7 / 100000 niños por año ${ }^{[1]}$. El fenómeno Kasabach-Merritt (FKM) es una de las complicaciones del HEK, caracterizado por coagulopatía de consumo, trombocitopenia y alta mortalidad ${ }^{[2]}$.

La mayoría de los casos reportados son en niños menores de un año, predominantemente en los primeros seis meses de vida. Esta neoplasia generalmente se localiza en tronco y extremidades, lo que no excluye su presentación en otras zonas o con sintomatología diversa. Boccara et al., realizaron un reporte de 24 infantes afectados, de los cuales 15 presentaron lesiones equimóticas y alteraciones de la coagulación desde el nacimiento, mientras que el resto dentro de los primeros 6 meses ${ }^{[3]}$.

Dentro de los esquemas terapéuticos reportados, se ha encontrado desde el uso de esteroides sistémicos, antiplaquetarios, hasta citostáticos como vincristina, entre otros ${ }^{[4,5]}$. Sin embargo, a pesar de las distintas opciones de tratamiento, la respuesta terapéutica no suele ser satisfactoria, existiendo casos refractarios al tratamiento, lo que dificulta lograr un consenso en el manejo y apertura la posibilidad de seguir investigando sobre nuevas opciones.

El sirolimus es un fármaco inmunosupresor comúnmente empleado en pacientes postrasplantados, para la prevención del rechazo al aloinjerto. En los últimos años, se ha probado su empleo en el tratamiento de anomalías vasculares complicadas, especialmente en casos de HEK refractarios, en quienes se ha obtenido respuesta favorable ${ }^{[5,6]}$.

El objetivo de este reporte es mostrar la experiencia del tratamiento con sirolimus vía oral, en el caso de un paciente con HEK asociado a FKM, refractario al tratamiento habitual, con buena respuesta ante la administración de este fármaco.

\section{REPORTE DE CASO}

Lactante mujer de 4 meses, procedente de Lima, nacida por parto vaginal a término; ingresa el 13 de junio del 2016 a Emergencia del Instituto Nacional de Salud del Niño por cuadro clínico de una semana de duración, consistente en la aparición de una tumoración violácea en hemitórax izquierdo, dolorosa a la palpación, que aumenta de volumen en forma progresiva, comprometiendo miembro superior y tornándose indurada (Figura 1). No presenta enfermedades previas ni antecedentes familiares.

Al ingreso se encontró hematocrito (Hto): $28 \%$; conteo de plaquetas: 52 000/ $\mathrm{mm}^{3}$; tiempo de protrombina (TP): 19,3 seg, INR: 1,6; tiempo de tromboplastina parcial activado (TTPa): 43,5 seg; y fibrinógeno: $66,9 \mathrm{mg} / \mathrm{dl}$. Se la hospitalizó en el servicio de Dermatología con el diagnóstico de hemangioendotelioma kaposiforme y fenómeno de Kasabach Merritt, iniciándose tratamiento con metilprednisolona $3 \mathrm{mg} / \mathrm{kg} / \mathrm{día}$ IV.

Veinticuatro horas después de ser hospitalizada, pasó a la Unidad de Cuidados Intensivos (UCI) por alto riesgo de sangrado y persistencia de alteración hematológica y de coagulación. En UCl presentó la siguiente analítica: leucocitos: 10730/mm (abastonados: $0 \%$, neutrofilos: $60 \%$ ); Hto: $29 \%$; hemoglobina: $9,8 \mathrm{~g} / \mathrm{dl}$; conteo de plaquetas $47000 / \mathrm{mm}^{3}$; TP 19,9 seg; INR 1,7; TTPa 38,5 seg; tiempo de trombina (TT): 42 seg; fibrinógeno 45,9 mg/dl; y dímero $\mathrm{D} 36,9 \mathrm{mg} / \mathrm{ml}$.

Adicionalmente, se realizó un examen de lámina periférica, en el cual se encontró policromatofilia $1+$, anisocitosis $1+$, microcitosis $1+$, poiquilocitosis $2+$, hematíes fragmentados y macroplaquetas. Cabe destacar que los exámenes de glicemia, pruebas de función hepática y proteínas totales presentaron valores normales y el examen de proteína $\mathrm{C}$ reactiva (PCR) fue negativo.

Al día siguiente, con estos resultados, se inició tratamiento con vincristina IV, a una dosis de $0,05 \mathrm{mg} / \mathrm{kg} / \mathrm{semanal}$. Se realizó una radiografía de tórax que evidenció aumento de volumen de partes blandas del hemitórax izquierdo, con compromiso pleural y mediastínico. En forma similar, una ecografía doppler de partes blandas

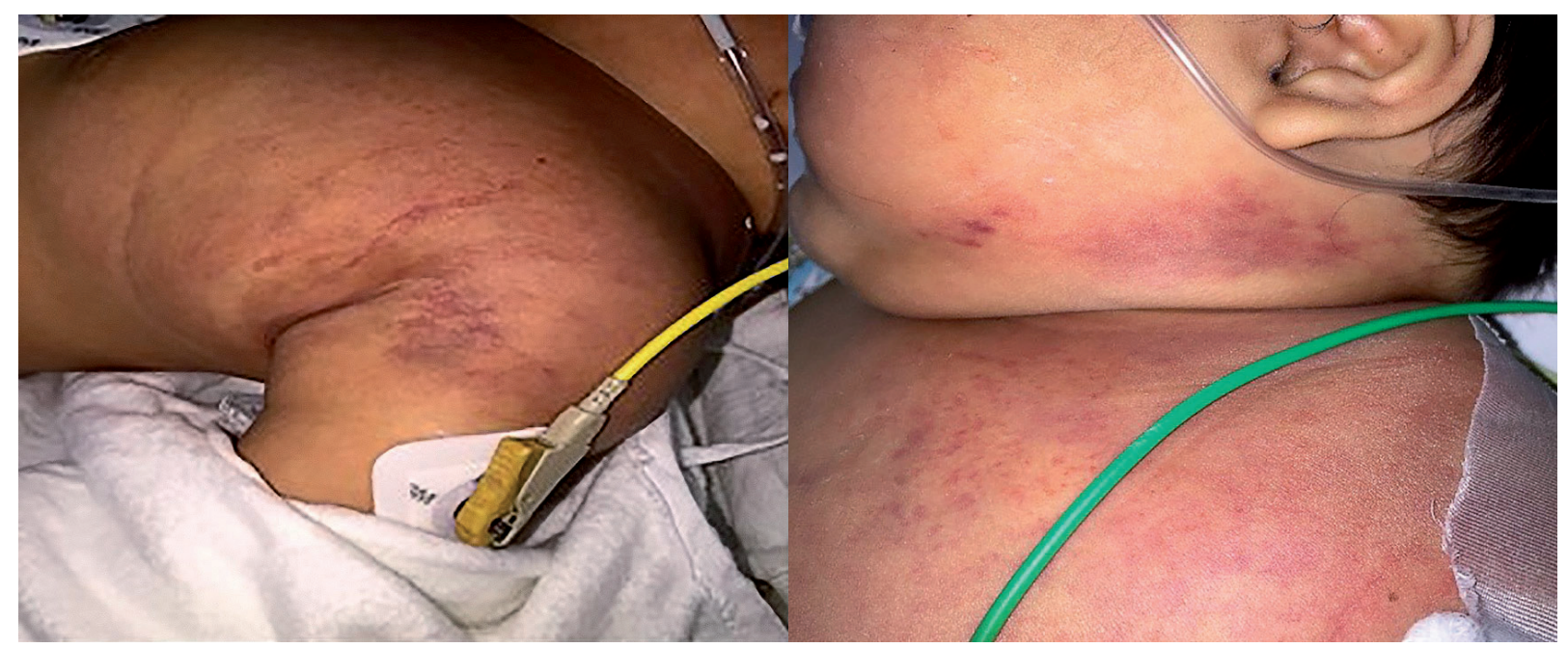

Figura 1. Tumoración violácea e indurada en región superior del hemitórax izquierdo que compromete miembro superior ipsilateral y región cervical. 
mostró lesión tumoral vascular con extensión a paquetes musculares. En base a ello, se ordenó una tomografía espiral multicorte (TEM) a nivel torácico que muestra tumoración difusa de partes blandas en hombro izquierdo con extensión a mediastino y parénquima pulmonar, asociado a un proceso inflamatorio bronco parenquimal izquierdo con derrame y engrosamiento pleural (Figura 2).

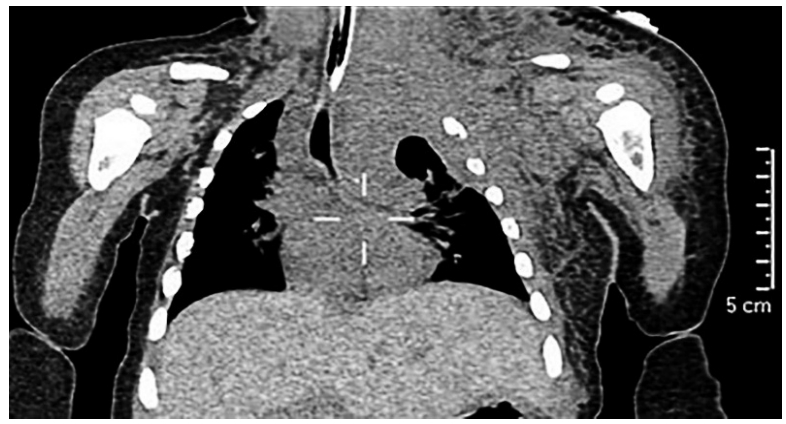

Figura 2. TEM torácica que muestra tumoración de aspecto vascular difuso de partes blandas extratorácicas en hombro izquierdo con extensión a mediastino, proceso inflamatorio broncoparenquimal izquierdo, derrame y engrosamiento pleural izquierdo.

Durante las primeras tres semanas se presentó aumento de volumen y extensión de la lesión, comprometiendo región cervical y mitad del antebrazo izquierdo. Se realizó una resonancia magnética nuclear (RMN) torácica que evidenció una lesión vascular infiltrativa de pared torácica izquierda que invade músculos intercostales e ingresa en cavidad torácica a nivel del ápex, extendiéndose hacia partes blandas de la región cervical, con signos de trombosis de venas del cuello. Con estos resultados se planteó la posibilidad diagnostica de síndrome de vena cava superior.

Se realizó una biopsia de la lesión, la que mostró células monocromáticas de aspecto fusiforme, extravasación de glóbulos rojos y formación de conglomerados celulares, lo que confirmó el diagnóstico de HEK (Figura 3). Ante la posibilidad de neoplasia maligna, se realizó inmunohistoquímica, resultando negativa.

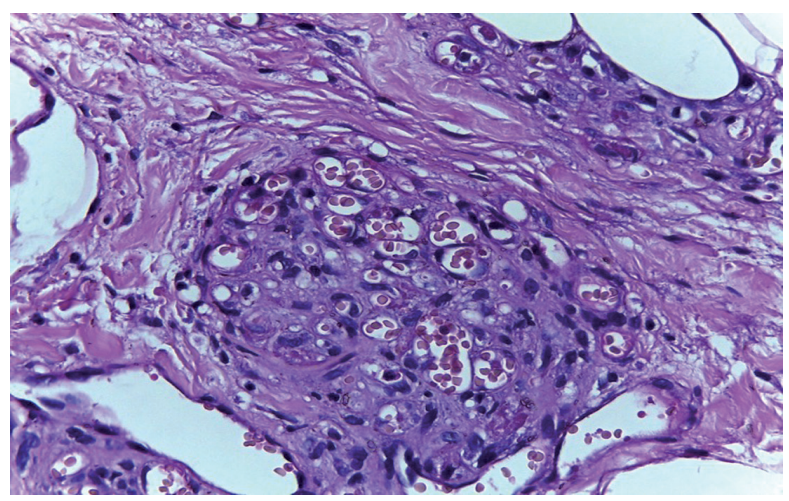

Figura 3. Histopatología de la tumoración compatible con hemangioendioteloma kaposiforme (40x). Se observan células monocromáticas de aspecto fusiforme que envuelven vasos capilares con extravasación de glóbulos rojos, formando conglomerados celulares.
La paciente presentó evolución tórpida, con desaturación, cianosis y dificultad respiratoria, hasta que 30 días después de su ingreso a $\mathrm{UCl}$, presentó un paro respiratorio, requiriendo ventilación mecánica. A los 15 días del ingresar a ventilación mecánica presentó fracaso en extubación, por probable obstrucción de vía aérea secundaria a infiltración tumoral. Por ello, se realizó una video-fibrobroncoscopía evidencia compresión extrínseca moderada a severa de $2 / 3$ distales de bronquio principal izquierdo.

Durante el primer mes de hospitalización recibió cinco pulsos de metilprednisolona y vincristina semanales, manteniéndose con propranolol $3 \mathrm{mg} / \mathrm{kg} /$ día, prednisona $2 \mathrm{mg} / \mathrm{kg} /$ día y ácido acetil salicílico $10 \mathrm{mg} / \mathrm{kg} /$ día, luego de la suspensión de vincristina, sin respuesta favorable.

Por persistencia de evolución clínica desfavorable, plaquetopenia y consumo de factores de coagulación en grado severo, se reinició, luego de 3 semanas de suspensión, tratamiento con vincristina y se agregó sirolimus a $0,8 \mathrm{mg} / \mathrm{m}^{2} /$ dosis, cada $12 \mathrm{~h}$.

Una semana después del inicio del sirolimus, se realizó un dosaje sérico que resulta en valores normales $(7,9 \mathrm{ng} / \mathrm{ml}$; VN3 - $18 \mathrm{ng} / \mathrm{ml}$ ), por lo que se incrementó dosis a $1 \mathrm{mg} / \mathrm{m}^{2} / \mathrm{do}$. A la segunda semana de tratamiento con sirolimus, se evidenció regresión parcial de la lesión y mejoría de valores hematológicos y de coagulación. A los 20 días del tratamiento, se realizó la extubación, con los siguientes controles analíticos: conteo de plaquetas: 162 000/mm³ H Ho 31\%; TP 13,5"; INR 1,2; TTPa 30,3"; TT 24"; y fibrinógeno $119 \mathrm{mg} / \mathrm{dl}$.

Durante la cuarta semana de tratamiento con sirolimus se suspendió la administración de vincristina. Egresa de $\mathrm{UCl}$ a los 12 días postextubación, manteniendo tratamiento diario con sirolimus (nivel sérico previo al alta: $2,7 \mathrm{ng} / \mathrm{ml}$ ). Este tratamiento persistió al alta hospitalaria y se recomendó seguimiento por consultorio externo. A los 6 meses del tratamiento con sirolimus, se encuentra una notable regresión de la lesión (Figura 4).

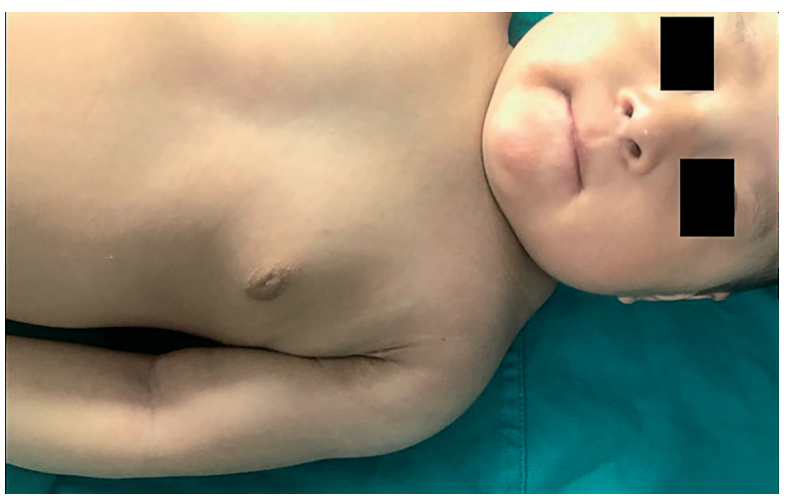

Figura 4. Imagen tomada a los 6 meses de tratamiento con sirolimus que muestra remisión de la tumoración vascular en región superior del hemitórax izquierdo. Aún se observa una pequeña lesión violácea remanente en área afectada. 


\section{DISCUSIÓN}

El HEK es un tumor vascular localmente agresivo de la infancia, con características comunes entre hemangiomas capilares y sarcoma de Kaposi ${ }^{[1,7,8]}$. Constituye un cuadro clínico grave, con una mortalidad promedio del $24 \%$, que puede ser mayor en pacientes con menos de 6 meses de edad ${ }^{[4]}$.

La presentación clínica usualmente se inicia con la aparición de una lesión cutánea de crecimiento rápido e invasivo, de localización variable; que se caracteriza por presentar, a la histología, proliferación endotelial que invade piel, tejido adiposo, muscular e incluso puede ocasionar destrucción ósea ${ }^{[6]}$. Es importante considerar este aspecto, ya que el compromiso óseo presentado por el HEK puede ser radiográficamente indistinguible del rabdomiosarcoma o fibromatosis agresiva ${ }^{[9]}$. En este caso clínico, no se observó infiltración ósea en los exámenes de imágenes; sin embargo, los resultados de la RMN evidencian la alta capacidad infiltrativa de esta lesión maligna.

El HEK tiene un curso impredecible y se considera que aquellos tumores con infiltración visceral conforman lesiones extensas e irresecables, por ende, con un mal pronóstico ${ }^{[2,9]}$. La afectación visceral es extremadamente rara y se encuentra asociada al fenómeno de Kasabach Merritt (FKM) y linfangiomatosis ${ }^{[9]}$. El caso presentado nos muestra que si bien este fenómeno es raro en la epidemiologia del HEK, debe siempre ser considerado ante la sospecha diagnostica, por su alta mortalidad y mal pronóstico.

El FKM es una entidad común asociada a diversos tumores vasculares y no es exclusiva del HEK. Se caracteriza por la presentación de una trombocitopenia severa, anemia hemolítica microangiopática, y coagulopatía de consumo ${ }^{[2,10]}$. Este fenómeno consiste en la captura de plaquetas y factores de coagulación a través de la proliferación anormal del endotelio vascular, que puede progresar a una coagulación intravascular diseminada (CID) ${ }^{[1,7]}$. May J et al. reportaron el caso de una niña de 4 semanas con una lesión equimótica en tronco, que luego se complicó con trombocitopenia severa y alteración franca de la coagulación, además de elevación del dímero D1 ${ }^{[1]}$. Esta presentación es similar al caso presentado en este reporte, por lo que se reitera la necesidad de detectar todo cuadro clínico similar e incluir en las presunciones diagnosticas posibles al HEK.

Como en toda lesión neoplásica, el diagnóstico definitivo lo brinda el examen histopatológico, producto de la realización de una biopsia, que revela presencia de nódulos y nidos de células fusiformes, atipia celular y escasa actividad mitótica ${ }^{[4,6]}$. No obstante, muchas veces no es posible realizar este procedimiento debido al riesgo de hemorragia, por lo que el médico tratante debe brindar especial importancia a la delimitación del tumor y su caracterización con apoyo de exámenes de imágenes.

Aunque el tratamiento definitivo del HEK sería la escisión quirúrgica completa, esto depende de la ubicación y extensión del tumor, por lo que por su rápida infiltración tisular o su asociación a FKM, no todas pueden ser resecadas ${ }^{[9]}$. Esta situación fue la presentada en nuestro caso clínico, y es ahí en donde actualmente no existe consenso sobre las opciones terapéuticas a brindar. Usualmente se acude a tratamientos farmacológicos, basados en ciclos de esteroides sistémicos y vincristina ${ }^{[11]}$. Adicionalmente, se debe considerar que la prescripción de transfusiones de hemoderivados debe restringirse a casos de hemorragia aguda severa o cirugía ${ }^{[6]}$.

El tratamiento con vincristina se suele recomendar para el HEK que no responde a corticoides; aunque también se han publicado experiencias con esquemas combinados ${ }^{[10]}$. Boccara et al. publicaron 24 casos de infantes dentro los primeros 6 meses de vida, con diagnóstico de HEK complicados con FKM, de los cuales el $62 \%$ respondieron al uso de Vincristina, mientras que sólo el $13 \%$ al uso de corticoides ${ }^{[3]}$. Adicionalmente, se halló que la combinación vincristina/corticoides normalizó el recuento plaquetario en aproximadamente 5 semanas ${ }^{[3]}$.

Por otro lado, Drolet et al., recomiendan la terapia inicial con vincristina IV $0,05 \mathrm{mg} / \mathrm{kg} / \mathrm{semanal}$ y prednisolona $2 \mathrm{mg} / \mathrm{kg} /$ día VO o metilprednisolona IV a dosis de $1,6 \mathrm{mg} / \mathrm{kg} / \mathrm{día}^{[2]}$. El esquema terapéutico que se aplicó en nuestro caso, además de vincristina, incluyó el uso de propranolol y ácido acetilsalicílico (aspirina) durante tres semanas sin obtener mejoría, aunque el uso de estos últimos es discutido ${ }^{[1]}$.

Sirolimus (rapamicina) es un inmunosupresor, inhibidor mTOR (mammalian target of rapamycin), que suprime la vasculogénesis y angiogénesis por lo que ha sido usado en casos refractarios ${ }^{[10]}$. En el 2016, Adams et al. presentaron aproximadamente 60 casos de pacientes con anomalías vasculares refractarias a la terapia convencional con corticoides, en quienes se observó una respuesta favorable a sirolimus, con buenos resultados al primer año de tratamiento ${ }^{[12]}$.

Así mismo, Tan X et al. reportaron una serie de casos de ocho lactantes con tumores vasculares asociados a FKM refractarios a corticoides, en el cual, siete de ellos respondieron positivamente al tratamiento con sirolimus, observándose mejoría a la semana de inicio del tratamiento ${ }^{[13]}$. En este caso, el tratamiento con sirolimus se inició con dosis de 0,8 mg/m2/do, c/12 h, similar a lo utilizado en un reporte de Sakata et al. donde se observó normalización de parámetros hematológicos y disminución de la masa tumoral a los tres meses de iniciado el tratamiento ${ }^{[14]}$. Luego mejoría de valores hematológicos a las dos semanas y disminución del volumen tumoral al mes del tratamiento.

Otros estudios han encontrado resultados coherentes al presentado en este caso. Por ejemplo, en una serie de casos publicada en el 2014 por Kai Li et al. todos los pacientes fueron tratados con al menos dos fármacos previo al tratamiento con sirolimus, observándose el inicio de la respuesta a los cinco días; mientras que la estabilización del recuento plaquetario ocurrió a los 15 días, en quienes recibieron sirolimus asociado a otros fármacos; y en 1 -2 meses, en los casos de monoterapia con sirolimus ${ }^{[5]}$. 
Así mismo, se debe considerar la importancia de un monitoreo estricto de los valores séricos de sirolimus, ajustándose las dosis de acuerdo a los resultados obtenidos en estos exámenes. El reporte de Jahnel et al. presentó dos casos, el primero recibió vincristina $0,5 \mathrm{mg} / \mathrm{m}^{2}$, previa no respuesta a corticoides y a un pulso de ciclofosfamida; y el segundo, recibió una sola dosis de ciclofosfamida $300 \mathrm{mg} / \mathrm{m}^{2}$, corticoides en pulsos y vincristina 0,5 $\mathrm{mg} / \mathrm{m}^{2} / \mathrm{semanal}$ durante 11 semanas, requiriendo sirolimus vía oral a $0,1 \mathrm{mg} / \mathrm{kg} /$ día dividido en 2 dosis; mostrándose la importancia del ajuste según nivel sérico (objetivo: 10-15 ng/ml) ${ }^{[15]}$.

Otra controversia que puede surgir es el tiempo de tratamiento con sirolimus, necesario para mostrar efectos sostenidos. Los reportes encontrados mencionan intervalos bastante amplios, entre 1-12 meses ${ }^{[12]}$ o 8-14 meses ${ }^{[15]}$. En nuestro reporte, la paciente sigue recibiendo este tratamiento, 12 meses después del inicio, lo que es coherente con los intervalos mostrados por reportes previos.

Un factor a considerar en la decisión de la prescripción y el sostenimiento del tratamiento con sirolimus, es la aparición de algunos efectos secundarios que pueden ser transitorios y tolerables, como hiperlipemia, mucositis, hipertensión arterial y elevación de transaminasas ${ }^{[6,11]}$. Sin embargo, se ha descrito incremento del riesgo de desarrollar tumores tipo linfomas, e infecciones, por lo que se recomienda profilaxis para Pneumocystis jirovecii ${ }^{[15]}$.

La paciente obtuvo mejoría en un tiempo relativamente corto, evidenciado con la normalización plaquetaria, exámenes de coagulación y disminución evidente del tumor; sin evidencia de efectos adversos durante la administración del medicamento. Sin embargo, es importante reiterar que tanto la duración del tratamiento, como el seguimiento de posibles efectos adversos, solo es posible instaurando un control periódico estricto.

No se ha encontrado casos reportados en nuestro país sobre el uso de sirolimus en el tratamiento del KEH refractario con FKM severo. Aunque la bibliografía consultada lo sugiere entre otros medicamentos, se requieren mayores estudios para protocolizar el tratamiento de pacientes con HEK, para lo cual este reporte podría aportar información importante a tener en cuenta al momento de diseñar los mencionados estudios.

Contribución de los autores: DGP y JTD participaron en la concepción y el diseño del manuscrito, recolección, análisis e interpretación de los datos, redacción del manuscrito y aprobación del artículo enviado. FVV y RLP participaron en la concepción del manuscrito, su revisión crítica y aprobaron el artículo enviado. Todos los autores se responsabilizan de cualquier aspecto relacionado al manuscrito.

\section{Fuente de financiamiento: Autofinanciado}

Conflicto de interés: Los autores declaran no tener ningún conflicto de interés

\section{REFERENCIAS BIBLIOGRÁFICAS}

1. May J, Sadri A, Shanks L, Liew SH, Pizer B. Kaposiform haemangioendothelioma (KHE): Not just a bruise. JPRAS Open. 2014;1-2:1-5.

2. Drolet BA, Trenor CC, Brandão LR, Chiu YE, Chun RH, Dasgupta R, et al. Consensus-derived practice standards plan for complicated Kaposiform hemangioendothelioma. J Pediatr. 2013;163(1):28591.

3. Boccara O, Fraitag S, Lasne D, Fontaine J, Bughin V, Hamel-Teillac $D$, et al. Kaposiform Haemangioendothelioma-spectrum Lesions with Kasabach-Merritt Phenomenon: Retrospective Analysis and Long-term Outcome. Acta Derm Venereol. 2016;96(1):77-81.

4. Garcia-Monaco R, Giachetti A, Peralta O, Napoli N, Lobos P, Gioseffi L, et al. Kaposiform Hemangioendothelioma with Kasabach-Merritt Phenomenon: Successful Treatment with Embolization and Vincristine in Two Newborns. J Vasc Interv Radiol. 2012;23(3):417-22.

5. Kai L, Wang Z, Yao W, Dong K, Xiao X. Sirolimus, a promising treatment for refractory Kaposiform hemangioendothelioma. J Cancer Res Clin Oncol. 2014;140(3):471-6.

6. Rodríguez Z N, Benavides J P. Sirolimus (rapamicina) en pacientes con hemangioendotelioma kaposiforme: Caso clínico. Rev Chil Pediatría. 2013;84(5):537-44.

7. Atla B, Sudhakar PV, Rao N, Prasad U. Recurrent multifocal cutaneous Kaposiform hemangioendothelioma: A rare vascular tumor of infancy and childhood. Indian J Pathol Microbiol. 2016;59(3):392-4.

8. Mentzel T, Mazzoleni G, Dei Tos AP, Fletcher CD. Kaposiform hemangioendothelioma in adults. Clinicopathologic and immunohistochemical analysis of three cases. Am J Clin Pathol. 1997;108(4):450-5.

9. Kurian JJ, Kishore R, John TJ, Parmer H. A rare case of kaposiform hemangioendothelioma presenting as intussusception in a 4-month-old child without Kasabach-Merrit syndrome: A case report. J Indian Assoc Pediatr Surg. 2014;19(4):233-5.

10. Yuan S-M, Shen W-M, Chen H-N, Hong Z-J, Jiang H-Q. KasabachMerritt phenomenon in Chinese children: Report of 19 cases and brief review of literature. Int J Clin Exp Med. 2015;8(6):10006-10.

11. Tlougan BE, Lee MT, Drolet BA, Frieden IJ, Adams DM, Garzon MC. Medical management of tumors associated with KasabachMerritt phenomenon: an expert survey. J Pediatr Hematol Oncol. 2013;35(8):618-22.

12. Adams DM, Trenor CC, Hammill AM, Vinks AA, Patel MN, Chaudry G, et al. Efficacy and Safety of Sirolimus in the Treatment of Complicated Vascular Anomalies. Pediatrics. 2016;137(2):e20153257.

13. Tan X, Zhang J, Zhou S, Liu Z, Zhang T, Xia J. Successful management of steroid-resistant vascular tumors associated with the Kasabach-Merritt phenomenon using sirolimus. J Dermatol. 2018;45(5):580-3.

14. Sakata N, Suenobu S-I, Okano M, Ueda S, Kimura M, Takemura T. Impact of sirolimus treatment for refractory kaposiform hemangioendothelioma with exacerbation of the disease 10 years after initial diagnosis. Rare Tumors. 2018;10:2036361318776185.

15. Jahnel J, Lackner H, Reiterer F, Urlesberger B, Urban C. Kaposiform hemangioendothelioma with Kasabach-Merritt phenomenon: from vincristine to sirolimus. Klin Padiatr. 2012;224(6):395-7. 